\title{
Control of wall turbulence by spinning discs
}

\author{
Pierre Ricco ${ }^{1}$ and Stanislav Hahn ${ }^{2}$ \\ ${ }^{1}$ Department of Mechanical Engineering, The University of Sheffield, \\ Mappin Street, S1 3JD Sheffield, United Kingdom and \\ ${ }^{2}$ Honeywell Turbo Technologies, Turanka 100/1236, Brno, Czech Republic
}

(Dated: September 5, 2013)

Published as: Pierre Ricco, Stanislav Hahn, "Control of Wall Turbulence by Spinning Discs", Advanced Materials Research, Vol. 745, pag. 135-143, 2013

This article is an extension to a previous paper of ours. It summarizes the main findings and complements flow visualisations. An active technique for friction drag reduction in a turbulent channel flow is studied by direct numerical simulations. The flow is modified by the steady rotation of rigid flush-mounted discs, located next to one another on the walls. The effect of the disc motion on the friction drag is investigated at a Reynolds number of $R_{\tau}=180$, based on the friction velocity of the stationary-wall case and the half channel height. We compute a maximum drag reduction of $23 \%$ and a maximum net power saved of $10 \%$, calculated by taking into account the power needed to rotate the discs. The new Reynolds stress term induced by the disc rotation and generated by the velocity components of the time averaged flow is shown to be instrumental for drag reduction.

\section{INTRODUCTION}

Turbulent drag reduction represents one of the great challenges in current fluid mechanics research because of the vast energy savings that could be achieved in industrial applications. The need for lower fuel consumption and improved environmental sustainability has driven large efforts in the academic and industrial worlds to study new drag-reduction techniques.

A variety of open-loop active methods (for which energy is input to the system to modify the flow in a predetermined manner) has been studied recently. Spanwise wall oscillations have experienced a growing interest since first studied by Jung et al. (1992). Their time dependent wall forcing, $w=W \sin (2 \pi t / T)$, inspired Viotti et al. (2009) to convert the unsteady motion into a steady streamwise-dependent forcing, i.e. $w=W \sin (2 \pi x / \lambda)$. Although these methods are interesting for their large drag reduction (as high as $45 \%$ for unsteady forcing and $50 \%$ for steady wave), the net power saved ( $7.5 \%$ and $23 \%$ respectively), and as simple cases for studying the drag-reduction mechanism (Choi and Clayton, 2001; Ricco et al., 2012), their technological realization clearly remains a major challenge. One reason for this lies in the estimated oscillation frequency corresponding to maximum drag reduction extremely high, i.e. about $15 \mathrm{kHz}$ over the wing of a commercial aircraft at a cruise speed of $225 \mathrm{~m} / \mathrm{s}$ at $10 \mathrm{~km}$ above sea level.

A related novel device proposed by Keefe $(1997,1998)$ may instead offer interesting opportunities for industrial applications. This actuator consists of discs which are flush-mounted on a flat surface and rotate at constant angular velocity. To the best of our knowledge, neither experimental nor numerical studies exist on this type of flow. The effect of varying the disc diameter and rotational frequency on the near-wall turbulence were investigated through direct numerical simulations in the channel flow geometry. We herein focus on summarizing our main results, recently published in Ricco and Hahn (2013), and present some new previously unpublished flow visualisations.

\section{NUMERICAL PROCEDURES}

\section{A. Numerical solver of the Navier-Stokes equations}

A pressure-driven turbulent flow between infinite parallel flat plates at a low Reynolds number has been studied by direct numerical simulations. The open-source numerical code available on 


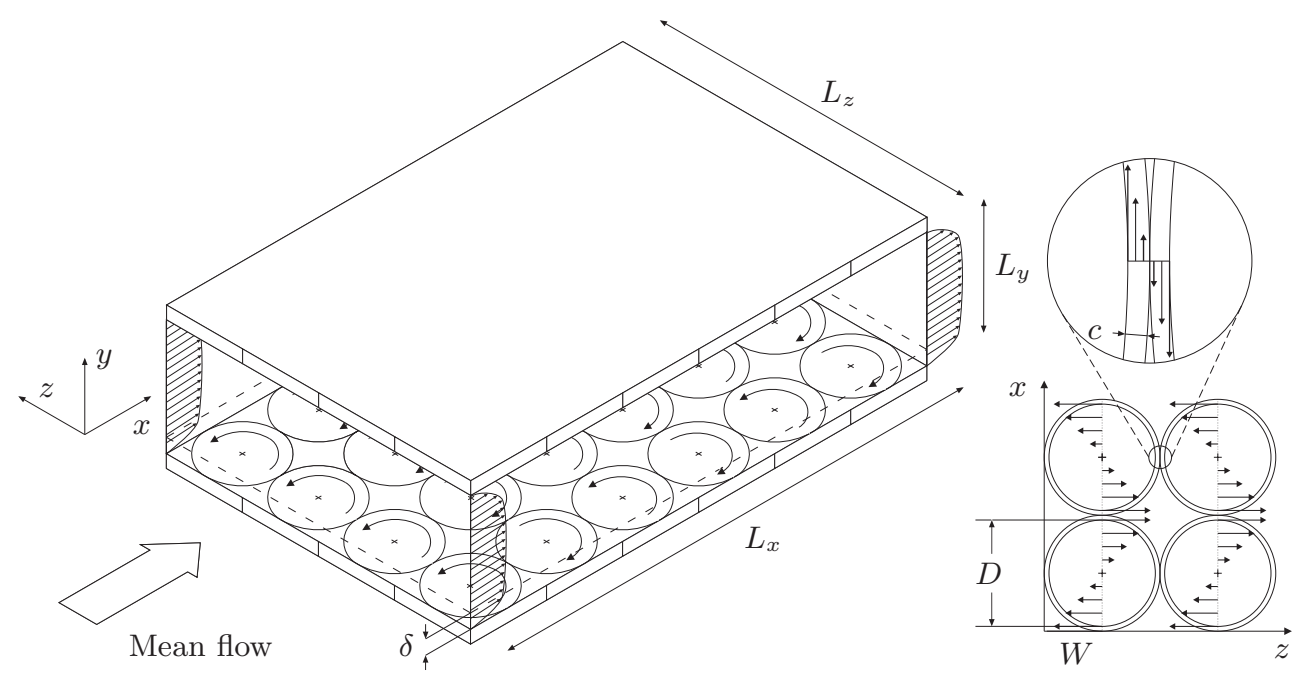

FIG. 1 Schematic of the turbulent channel flow with rotating discs. The graph on the right shows the $z$-component of the wall velocity along lines parallel to $x$ and passing through the disc centres.

the Internet (Gibson, 2006) has been modified to impose the disc motion. The code solves the incompressible Navier-Stokes equations in the channel flow geometry using Fourier series expansions along the streamwise $(x)$ and spanwise $(z)$ homogeneous directions, and Chebyshev polynomials along the wall-normal direction $(y)$. The numerical method is based on the Kleiser-Schumann algorithm (Kleiser and Schumann, 1980). The time-stepping algorithm (Ascher et al., 1995) is based on a third-order semi-implicit backward differentiation scheme (SBDF3). Dealiasing is performed at each time-step by setting to zero the upper one-third of the Fourier coefficients along the streamwise and spanwise directions. The calculations have been run in parallel using an OpenMP strategy on the computer cluster Iceberg at the University of Sheffield.

\section{B. Scaling of flow quantities and arrangement of discs}

Dimensional quantities are indicated by the symbol $*$. Lengths are scaled by $h^{*}$, the half channel height, velocities are scaled by $U_{p}^{*}$, the maximum centreline velocity of the laminar Poiseuille flow at the same mass flow rate, time is scaled by $h^{*} / U_{p}^{*}$, and pressure by $\rho^{*} U_{p}^{* 2}$, where $\rho^{*}$ is the density. Quantities scaled by these outer units are not marked by any symbol. The symbol + denotes scaling by the viscous inner units, i.e. by the kinematic viscosity $\nu^{*}$ and the friction velocity $u_{\tau}^{*}=\sqrt{\tau_{w}^{*} / \rho^{*}}$, where $\tau_{w}^{*}$ is the space- and time-averaged wall-shear stress.

The flow domain is shown in figure 1 . The discs have a diameter $D$ and rotate at a constant angular velocity $\Omega$ with tip velocity $W=\Omega D / 2$. Neighbouring discs along $x$ have opposite direction of rotation and the direction of rotation along rows in the $z$ direction is the same. The disc velocity is first assigned in the physical space and then transformed into the spectral space to create the Fourier mode representation. Dirichlet boundary conditions are imposed on the equations of motion of the modes.

A thin buffer annular region around each disc is simulated, as shown in figure 1 (right), and is described in more detail in Ricco and Hahn (2013). The simulation of this region is useful because it provides an idealized representation of the gap between a disc and the stationary wall (which would be present in a laboratory), and because, as the wall velocity is now continuous, the Gibbs phenomenon generating spurious oscillations is strongly reduced is a sufficient number of Fourier modes is used. 


\section{Numerical parameters and averaging procedures}

The simulations have been performed at a constant mass flow rate and at a Reynolds number $R_{p}=U_{p}^{*} h^{*} / \nu^{*}=4200$. The stationary-wall friction Reynolds number is $R_{\tau, s}=u_{\tau}^{*} h^{*} / \nu^{*}=179.8$ (where the subscript $s$ indicates stationary-wall conditions). The dimensions of the computational domain are $L_{x}=6.79 \pi, L_{y}=2$, and $L_{z}=2.26 \pi$, i.e. $L_{x}^{+}=3840, L_{y}^{+}=360$, and $L_{z}^{+}=1280$ (viscous inner units of the stationary-wall case are used henceforth in this section, unless otherwise stated). The numbers of Fourier modes are $N_{x}=334$ along $x$ and $N_{z}=222$ along $z$, providing spatial resolutions of $\Delta x^{+}=11.5$ and $\Delta z^{+}=5.75$. Larger box sizes have been used for $D=5.07$ and $10.35, L_{x}=6.79 \pi$, $L_{z}=3.4 \pi\left(N_{z}=334, L_{z}^{+}=1926\right)$, and for $D=6.83, L_{x}=9.05 \pi, L_{z}=2.26 \pi \quad\left(N_{x}=446, L_{x}^{+}=5118\right)$. The number of modes has been adjusted to keep the same resolution for all cases. Along $y, N_{y}=129$ collocation points have been used along a stretched grid with resolution of $\Delta y_{\min }^{+}=0.054$ near the wall and $\Delta y_{\max }^{+}=4.42$ at the centreline. The time step varied between $\Delta t_{\min }^{+}=0.008$ and $\Delta t_{\max }^{+}=0.08$.

By defining

$$
\langle\mathbf{f}\rangle \equiv \frac{1}{L_{x} L_{z}} \int_{0}^{L_{z}} \int_{0}^{L_{x}} \mathbf{f} \mathrm{d} x \mathrm{~d} z, \quad \overline{\mathbf{f}} \equiv \frac{1}{t_{f}-t_{i}} \int_{t_{i}}^{t_{f}} \mathbf{f} \mathrm{d} t,
$$

where $t_{i}$ and $t_{f}$ denote the start and finish averaging time, the flow field is expressed as $\mathbf{u}(x, y, z, t)=\mathbf{u}_{\mathbf{m}}+\mathbf{u}_{\mathbf{d}}+\mathbf{u}_{\mathbf{t}}$, where $\mathbf{u}_{\mathbf{m}}(y)=\left\{u_{m}, 0,0\right\}=\langle\overline{\mathbf{u}}\rangle$ is the mean flow, $\mathbf{u}_{\mathbf{d}}(x, y, z)=$ $\left\{u_{d}, v_{d}, w_{d}\right\}=\overline{\mathbf{u}}-\mathbf{u}_{\mathbf{m}}$ is the disc flow, and $\mathbf{u}_{\mathbf{t}}(x, y, z, t)$ represents the turbulent fluctuations. The drag reduction is defined as $\mathcal{R}(\%) \equiv 100\left[1-u_{m}^{\prime}(0) / u_{m, s}^{\prime}(0)\right]$, where the prime denotes differentiation with respect to $y$. The bulk velocity is defined as $U_{b} \equiv \int_{0}^{1} u_{m}(y) \mathrm{d} y$. A total of 50 cases were run, for which $W$ and $D$ were varied independently in the parameter range $0.064 \leq W \leq 0.77,0.84 \leq D \leq 10.35$. A summary of resolution checks can be found in Ricco and Hahn (2013).

\section{RESULTS AND DISCUSSION}

\section{A. Turbulent drag reduction and net power saved as a functions of $D$ and $W$}

Figure 2 shows a three-dimensional map of $\mathcal{R}\left(D^{+}, W^{+}\right)(\%)$, where the size of the circles is proportional to the absolute value of $\mathcal{R}$. For fixed $W$, drag reduction occurs when $D$ is larger than a threshold, while the drag increases for smaller $D$ (the shaded areas denote drag-increase cases). The threshold $D$ for drag reduction (indicated by the dashed line bounding the shaded areas), the optimal $D$ at fixed $W$ and the optimal $W$ at fixed $D$ (denoted by black dots and open circles respectively in figure 2) all increase with $W$. An overall maximum $\mathcal{R}=22.9 \%$ is computed for $D=5.07$ and $W=0.39\left(D^{+}=801\right.$ and $\left.W^{+}=10.2\right)$.

As the disc-flow technique is active, power is used for rotating the discs against the viscous resistance of the fluid. The power $\mathcal{P}_{s p, t}(\%)$ is the percentage of the power spent $\mathcal{W}$ to rotate the discs with respect to the power $\mathcal{P}_{x}$ utilised to drive the flow along the streamwise direction in the stationary-wall case, i.e.

$$
\mathcal{P}_{s p, t}(\%) \equiv \frac{100 \mathcal{W}}{\mathcal{P}_{x}}=-\frac{100 R_{p}}{R_{\tau, s}^{2} U_{b}}\left\langle\left. u_{d}(x, 0, z) \frac{\partial u_{d}}{\partial y}\right|_{y=0}+\left.w_{d}(x, 0, z) \frac{\partial w_{d}}{\partial y}\right|_{y=0}\right\rangle
$$

which are found from the viscous work term in the kinetic energy equation (1-108) on page 71 in Hinze (1975). The net power saved $\mathcal{P}_{n e t}$ is also of interest, defined as the difference between the power saved thanks to the wall motion, which coincides with drag reduction at constant mass flow rate, and the power spent $\mathcal{P}_{s p, t}$, i.e. $\mathcal{P}_{\text {net }}(\%) \equiv \mathcal{R}(\%)-\mathcal{P}_{s p, t}(\%)$. The boxed numbers in figure 2 show that a positive $\mathcal{P}_{n e t}$ may occur for $W \leq 0.39$ and a maximum $\mathcal{P}_{n e t}=10.5 \%$ is computed for $D=5.07$ and $W=0.26\left(D^{+}=820, W^{+}=6.7, \mathcal{T}^{+}=386, \delta^{+}=9.1, \mathcal{R}=19.2 \%\right)$. The threshold diameter above which $\mathcal{P}_{\text {net }}$ is positive increases with $W$. The power $\mathcal{P}_{\text {net }}$ grows negative for small $D$ and large $W$ because both the drag and $\mathcal{P}_{s p, t}$ increase significantly. 


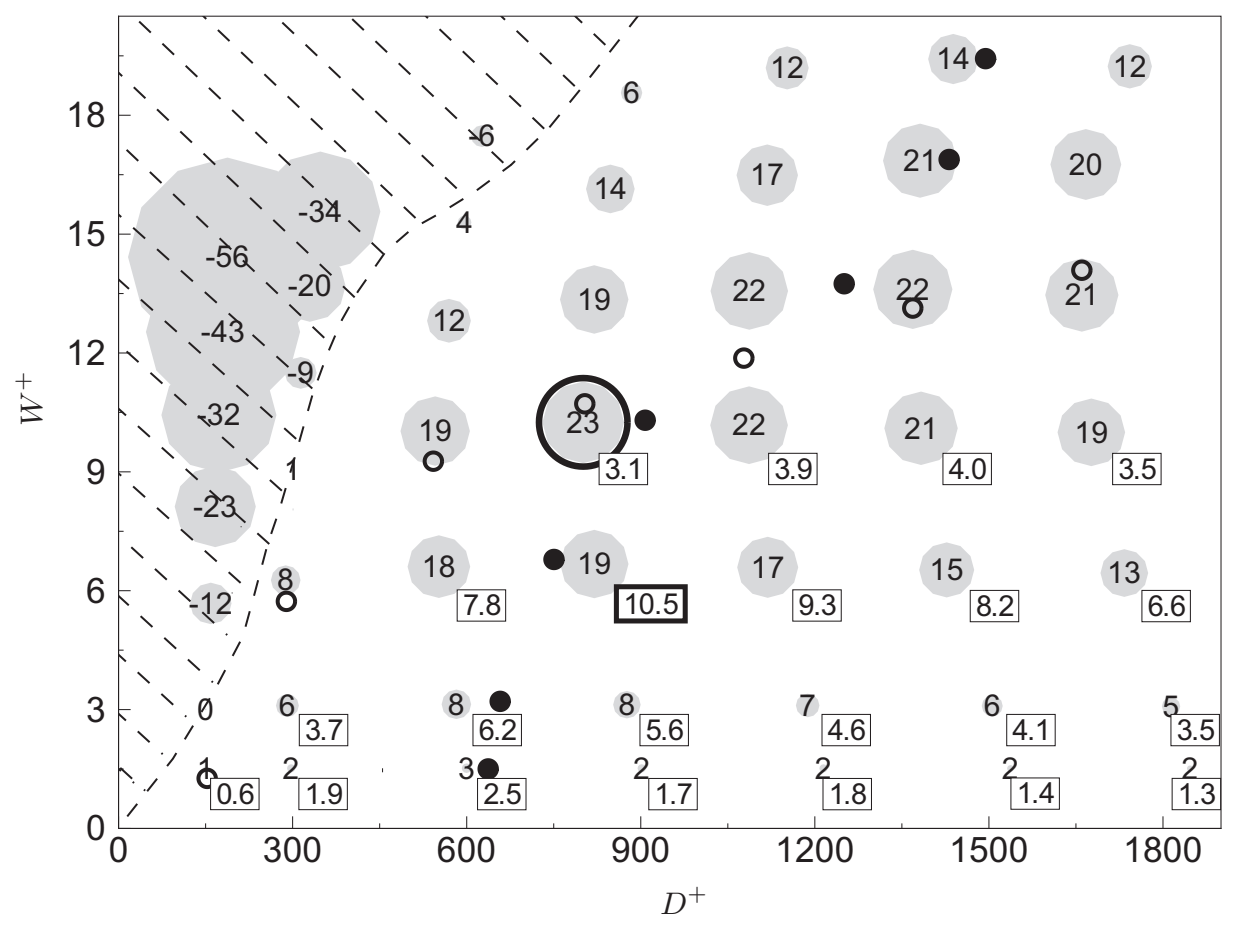

FIG. 2 Map of $\mathcal{R}\left(D^{+}, W^{+}\right)(\%)$. The size of the grey circles is proportional to the absolute value of $\mathcal{R}$. The hatched areas highlight the drag-increase cases and the zero- $\mathcal{R}$ lines (dashed) are found by linear data interpolation. The maximum $\mathcal{R}=22.9 \%$ is circled and the boxed values report the positive net power saved $\mathcal{P}_{n e t}(\%)$ (the thick box denotes the maximum $\mathcal{P}_{n e t}=10.5 \%$ ). The black dots indicate the estimated $D$ for maximum $\mathcal{R}$ at fixed $W$ and the open circles denote the estimated $W$ for maximum $\mathcal{R}$ at fixed $D$.

\section{B. Flow visualization of the time-averaged disc flow}

The case investigated in this and following section is for $D=3.38$ and $W=0.39\left(D^{+}=546\right.$ and $\left.W^{+}=10, \mathcal{R}=19.5 \%\right)$. Three-dimensional isosurfaces of $q^{+}\left(u_{d}^{+}, w_{d}^{+}\right) \equiv \sqrt{u_{d}^{+2}+w_{d}^{+2}}=2.3$, shown in figure 3 (top left), clearly show the time-averaged disc flow $\mathbf{u}_{\mathbf{d}}$ as near-wall circular patterns of thickness of about $10 \nu^{*} / u_{\tau}^{*}$. Streamwise-elongated structures appear over sections of stationary wall, where the shear generated by the tangential disc flow is largest because neighbouring discs along $z$ have opposite sense of rotation. Figure 3 (bottom left) shows that these structures have a round shape when observed on the $y-z$ plane at $x^{+}=0$, are centred at about $y^{+} \approx 40$, and are higher than the ring-shaped patterns as they reach about $y^{+} \approx 80$. Figure 3 (right) shows the contours of the same quantity observed on $y-z$ planes at three $x$ locations, $x^{+}=0,143.5$ and 287 . Only the bottom fifth of the domain is displayed along $y$ to clearly visualise the velocity distribution near the wall.

Contour plots of $u_{d}$ in $x-z$ planes are shown in figure 4. At $y^{+}=0$ the $u_{d}$ is highest at the disc edges, while the contour at $y^{+}=8$ reveals a wave-like flow pattern with wavelength $2 D+4 c$ following lines parallel to $x$ and passing through the disc centres. For $y^{+}>15$ the discs engender spanwisealternating streamwise bands of positive and negative $u_{d}$, i.e. of flow faster and slower than $u_{m}$ respectively. As shown by the contours at $y^{+}=27$ and $y^{+}=161$, the negative- $u_{d}$ bands become wider and the positive- $u_{d}$ bands become narrower as $y$ increases. The wide positive- $u_{d}$ bands are centred 

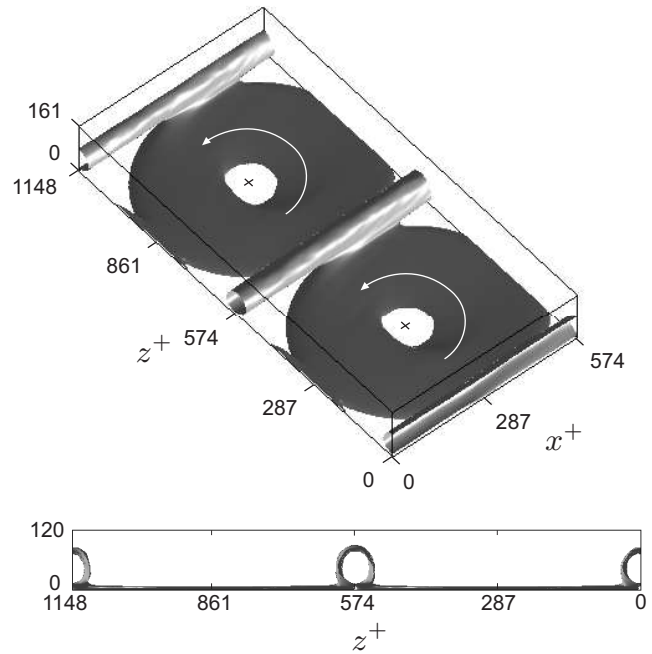

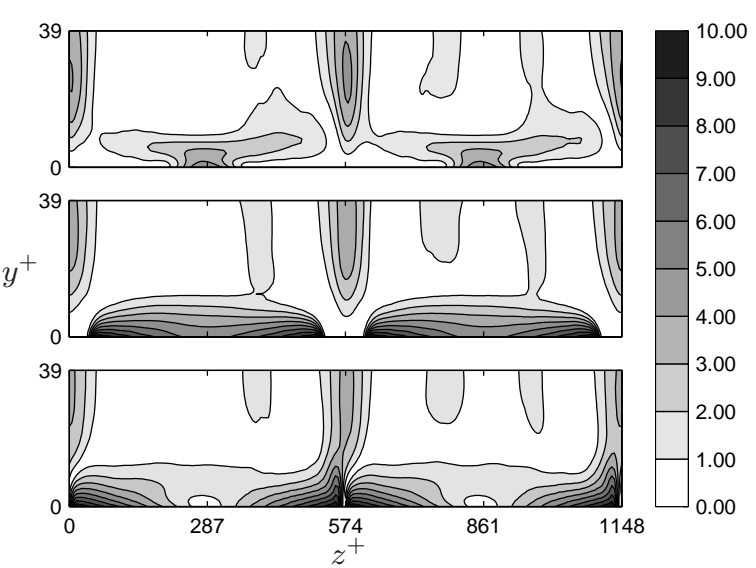
FIG. 3 Visualizations of the time-averaged disc flow $\mathbf{u}_{\mathbf{d}}$. Top left: isosurfaces of
$q^{+}\left(u_{d}^{+}, w_{d}^{+}\right) \equiv \sqrt{u_{d}^{+2}+w_{d}^{+2}}=2.3$, where one sixth of the domain is shown along $x$. Bottom left: the same quantity viewed normal to $y$ - $z$ plane. Right: Isosurfaces of the same quantity observed from the $y-z$ plane at $x^{+}=0, x^{+}=143.5$, and $x^{+}=287$, respectively.

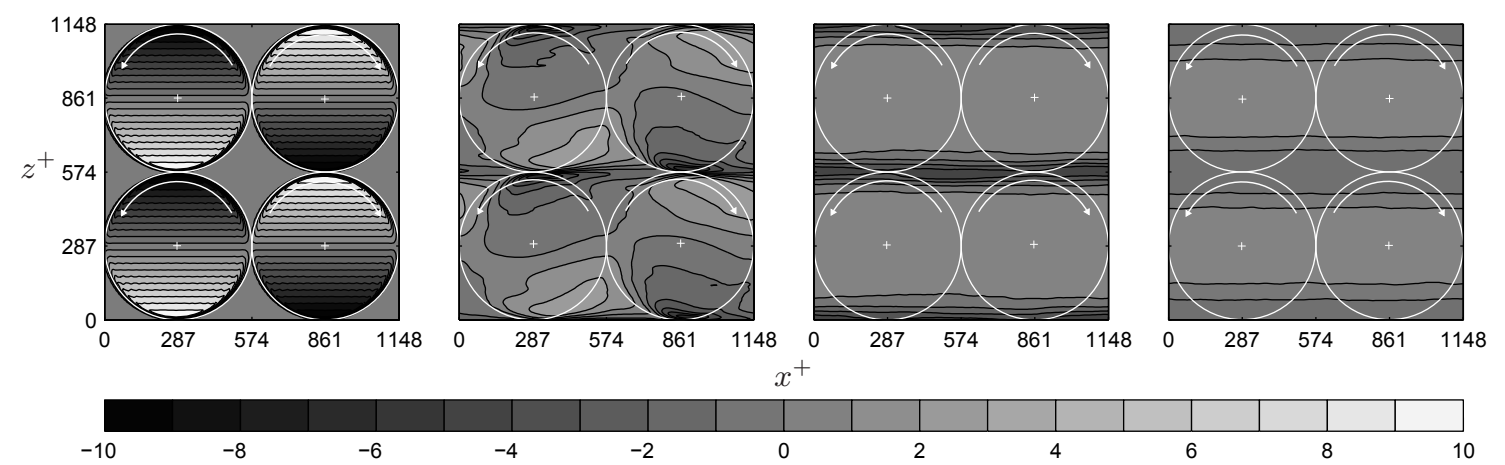

FIG. 4 Contour plot of $u_{d}^{+}$in the $x-z$ plane at $y^{+}=0$ (wall), $y^{+}=8, y^{+}=27$, and $y^{+}=161$ (centerline); one third of the domain is shown along $x$.

along discs centres, while the thinner and more energetic negative- $u_{d}$ bands are centred along lines tangent to the discs.

Figure 5 shows the contour plot of the scaled time-averaged wall-normal gradient of the streamwise velocity at the wall, $\left.2 \overline{\partial u / \partial y}\right|_{y=0} /\left(U_{b}^{2} R_{p}\right)$. Analogously to figure 4 the disc motion imposes a steady-wave pattern with a streamwise wavelength equal to $2 D+4 c$. The absolute value of the maximum negative streamwise wall-shear stress (about 0.02) is about three times larger than the spatial average, $C_{f}=6.64 \cdot 10^{-3}$. 


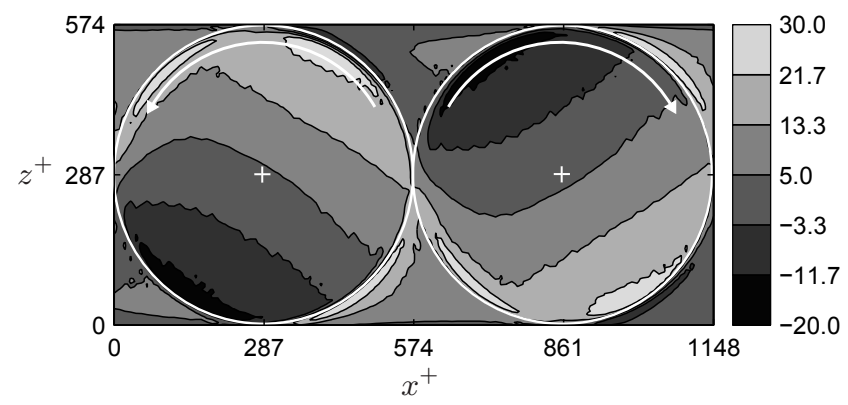

FIG. 5 Contour plot of time-averaged streamwise wall friction $2 \overline{\partial u / \partial y}_{y=0} /\left(U_{b}^{2} R_{p}\right)\left(\times 10^{3}\right)$. The skin-friction coefficient is $C_{f}=6.64 \cdot 10^{-3}$.
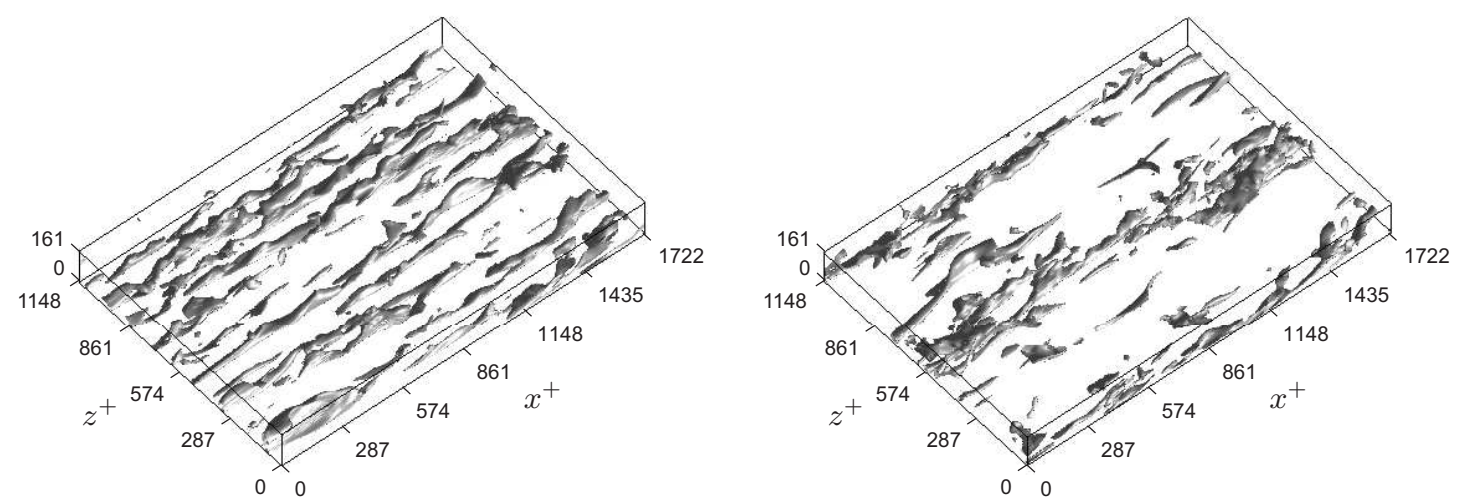

FIG. 6 Isosurfaces of $\operatorname{sgn}\left(u_{t}\right) q\left(u_{t}^{+}, w_{t}^{+}\right)=-3$ for stationary-wall (left) and disc-flow (right) cases.

\section{Turbulent flow visualizations and statistics}

Figure 6 shows instantaneous isosurfaces of $\operatorname{sgn}\left(u_{t}\right) q^{+}\left(u_{t}^{+}, w_{t}^{+}\right)=-3$ for stationary-wall (left) and disc-flow (right) conditions. The isosurfaces visualize the low-speed streaks, the intensity of which is reduced when they appear over the central part of the discs. They are instead less influenced when they are over stationary-wall sections between discs.

Figure 7 (left) presents the r.m.s. profiles of the components of $\mathbf{u}_{\mathbf{d}}$ (henceforth the r.m.s. of a quantity $a$ is defined as $\left.a_{r m s} \equiv \sqrt{\langle\overline{a a}\rangle}\right)$. The $u_{d, r m s}$ and $w_{d, r m s}$ profiles reduce from the wall $\left.\left(u_{d, r m s}^{+}(0)=w_{d, r m s}^{+}(0)=W^{+} \sqrt{(\pi / 3)\left[1+2 D^{+} /\left(2 c^{+}+D^{+}\right)\right.}\right] / 4=4.37\right)$ and overlap when $y^{+}<10$, while $u_{d, r m s}>w_{d, r m s}, v_{d, r m s}$ at higher locations. The $u_{d, r m s}$ profile reaches a local minimum at $y^{+} \approx 15$ $\left(u_{d, r m s}^{+} \approx 1.2\right)$ and a local maximum at $y^{+} \approx 35\left(u_{d, r m s}^{+} \approx 1.5\right)$. As shown by figure 3 (right), the minimum at $y^{+} \approx 15$ is the cut-off between the region where the disc-flow ring patterns exist near the wall and the region where the $x$-stretched bands appear at higher locations. The $w_{d, r m s}$ profile drops up to $y^{+} \approx 60$ to $w_{d, r m s}^{+} \approx 0.15$ and it matches the $v_{d, r m s}$ profile for $y^{+}>90$. Disc-flow turbulent boundary layer thickness $\delta$, a measure of the wall-normal diffusion of the time-averaged viscous effects generated by the disc rotation, is defined as $\delta \equiv-\left[u_{d, r m s}(0) / u_{d, r m s}^{\prime}(0)+w_{d, r m s}(0) / w_{d, r m s}^{\prime}(0)\right] / 2$, i.e. as the average of the $y$ locations obtained by the interception of the tangents at the wall of the $u_{d, r m s}$ and $w_{d, r m s}$ profiles with the $y$ axis (figure 7 , left). A minimum thickness is required for drag reduction; it increases from about $6 \nu^{*} / u_{\tau}^{*}$ at $W^{+}=4$ to about $7.5 \nu^{*} / u_{\tau}^{*}$ at $W^{+}=18$. The thickness $\delta^{+}=8.7$ corresponds to maximum drag reduction (refer to Ricco and Hahn 2013 for further details). 

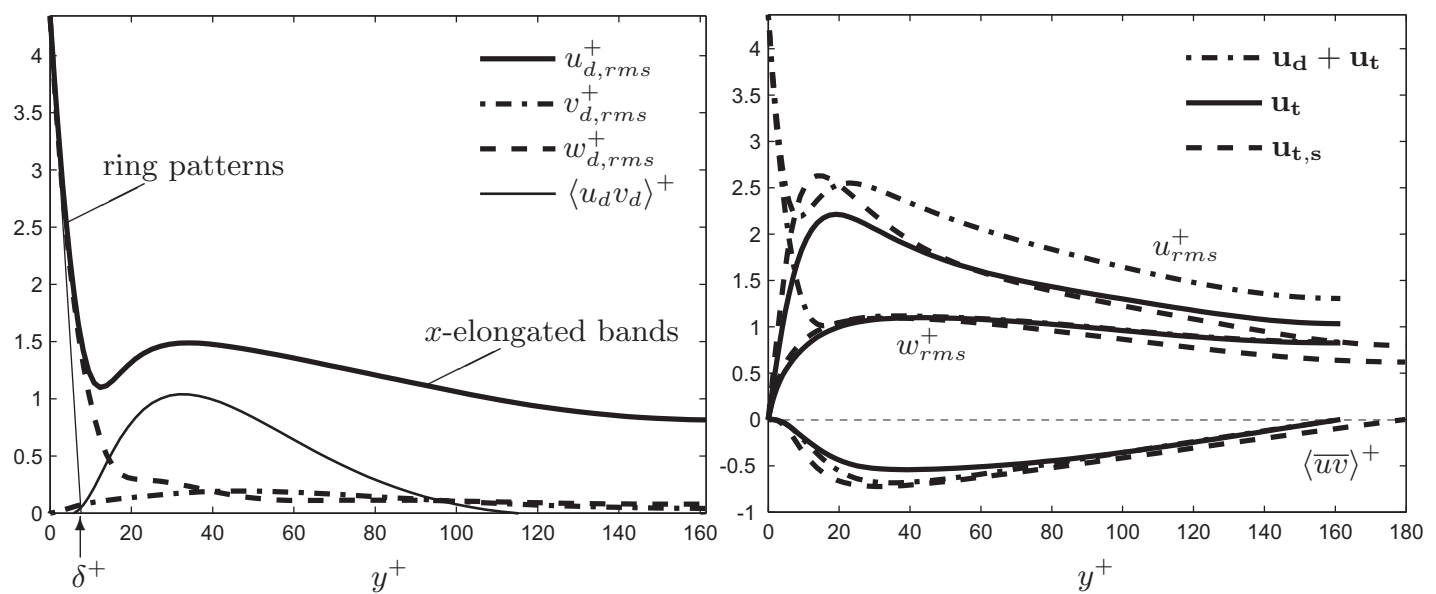

FIG. 7 Left: wall-normal profiles of r.m.s. of $\mathbf{u}_{\mathbf{d}}$ components and of $\left\langle u_{d} v_{d}\right\rangle^{+}$(the latter multiplied by a factor of 6); the disc-flow boundary layer thickness $\delta$ is shown. Right: wall-normal profiles of r.m.s. of velocity components and Reynolds stresses, where $u, v, w$ are indicated in the legend.

Figure 7 (right) shows the r.m.s. and Reynolds stresses $\langle\overline{u v}\rangle$ of $\mathbf{u}_{\mathbf{d}}+\mathbf{u}_{\mathbf{t}}$ (dash-dotted lines), $\mathbf{u}_{\mathbf{t}}$ (solid lines), and $\mathbf{u}_{\mathbf{t}, \mathbf{s}}$ (dashed line). The intensity of the turbulent fluctuations and the Reynolds stresses is lower near the wall when compared with the stationary-wall case, as for the oscillatingwall case (Choi et al., 1998). The $u_{r m s}$ is affected the most as the peak is reduced by about $15 \%$ and shifts upward from $y^{+} \approx 15$ to $y^{+} \approx 20$. The $\langle\overline{u v}\rangle$ peak decreases by about $30 \%$.

The FIK identity (Fukagata et al., 2002), modified to include the disc-flow effect, shows that the drag change is related to the disc-flow stresses $\left\langle u_{d} v_{d}\right\rangle$, shown in figure 7 (left), and to the modification of the turbulent stresses, $\left\langle\overline{u_{t} v_{t}}\right\rangle-\left\langle\overline{u_{t, s} v_{t, s}}\right\rangle$, as follows

$$
\mathcal{R}(\%)=100 \frac{R_{p} \int_{0}^{1}(1-y)\left[\left\langle\overline{u_{t} v_{t}}\right\rangle+\left\langle u_{d} v_{d}\right\rangle-\left\langle\overline{u_{t, s} v_{t, s}}\right\rangle\right] \mathrm{d} y}{U_{b}-R_{p} \int_{0}^{1}(1-y)\left\langle\overline{u_{t, s} v_{t, s}}\right\rangle \mathrm{d} y} .
$$

The drag reduction measured through $(2)$ is $\mathcal{R}=19.5 \%$ for $D=3.38$ and $W=0.39$, which agrees with the value computed by the wall-shear stress, also $\mathcal{R}=19.5 \%$. As suggested by figure 8 , the major contribution of $\left\langle u_{d} v_{d}\right\rangle$ comes from the $x$-stretched structures appearing between discs, while these stresses are of smaller magnitude over the discs. Along the dashed zero- $\mathcal{R}$ line in figure 2, both $\left\langle u_{d} v_{d}\right\rangle$ and $\left\langle\overline{u_{t} v_{t}}\right\rangle$ increase and balance each other as $W$ grows, leaving the drag unchanged.

\section{OUTLOOK FOR THE FUTURE}

Empirical correlations from Pope (2000) were used to estimate data for low-speed laboratory conditions and for three flows of technological interest for disc-flow parameters leading to $\mathcal{R}=20 \%$. Wind tunnel and water channel experiments may be realizable with contained costs as the diameter is of the order of $5-10 \mathrm{~cm}$ and the rotational frequency is about $35 \mathrm{~Hz}$ in air and less than $1 \mathrm{~Hz}$ in water (for $R_{\tau}=800$ for free-stream boundary layers and $R_{\tau}=180$ for channel flow).

The predicted quantities in flight conditions also look promising, as the diameter is about $6.7 \mathrm{~mm}$, the tip velocity is about $80 \mathrm{~m} / \mathrm{s}$, and the rotational frequency is about $3700 \mathrm{~Hz}$. A lower rotational frequency of $2230 \mathrm{~Hz}$ at the same diameter $\left(W^{*}=50 \mathrm{~m} / \mathrm{s}, W^{+}=6.5\right)$ guarantees $\mathcal{P}_{n e t}=8 \%$. The disc-flow parameters for turbulent flows over hulls of large-scale ships and over high-speed trains at the same flight-condition $R_{\tau}$ are also of interest. The disc diameters are similar to the ones in flight conditions $\left(D^{*}=6.5 \mathrm{~mm}\right.$ for ships and $D^{*}=8.1 \mathrm{~mm}$ for trains $)$, but the advantage is in the lower rotational rates, i.e. $W^{*}=3.5 \mathrm{~m} / \mathrm{s}$ and $f^{*}=170 \mathrm{~Hz}$ for ships, and $W^{*}=29 \mathrm{~m} / \mathrm{s}$ and about $f^{*}=1140 \mathrm{~Hz}$ 


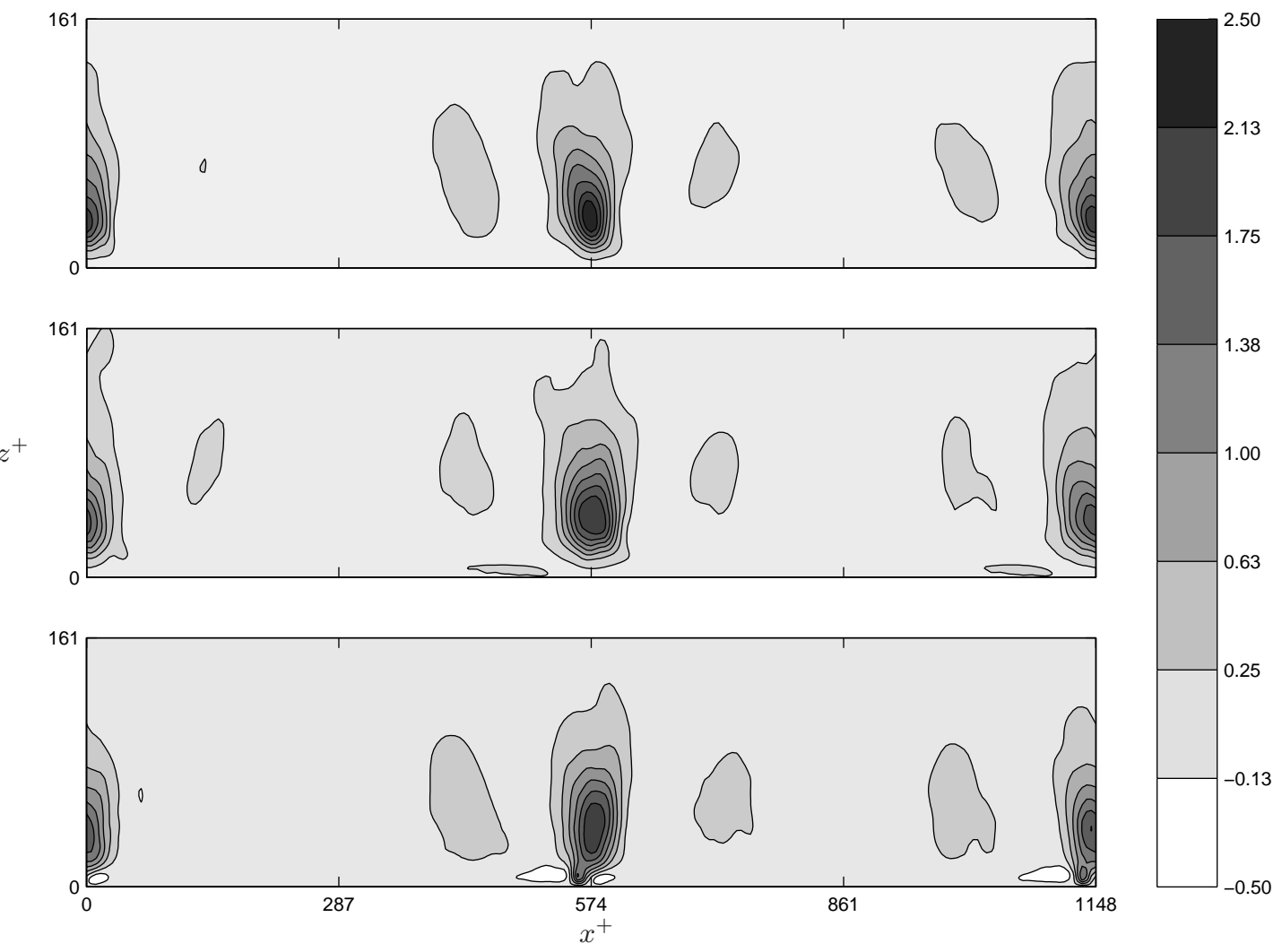

FIG. 8 Contour plot of $\left\langle u_{d} v_{d}\right\rangle^{+}$in the $y-z$ plane at $x^{+}=0, x^{+}=143.5$, and $x^{+}=287$, respectively.

for trains. The reader should refer to Ricco and Hahn (2013) for more details on the computation of these values and on the comparison with the original study by Keefe (1997). Rotation rates of $\mathcal{O}\left(10^{3}\right) \mathrm{Hz}$ may be obtained by commercially available electromagnetic motors which can be as small as $2 \mathrm{~mm}$ in diameter (Kuang-Chen Liu et al., 2010). Rotation rates of up to $\mathcal{O}\left(10^{4}\right)$ required for flight-conditions outlined above are achieveable by micromachined air turbines developed by Frechette et al. (2005).

Our results prove that forcing the near-wall turbulence at a scale which is much larger than that of the near-wall vortices (the optimal diameter is about five times the half channel height) is an effective method for drag reduction. This is in line with the works by Willis et al. (2010) and Sharma et al. (2011), which indicate that a near-wall body-force controller is most potent when acting on low-wavenumber structures.

The discs may offer some advantages over passive drag-reduction techniques, such as riblets and compliant surfaces, which have never been utilised in technological flow systems and have never led to amounts of drag reduction higher than $10 \%$ (Gad-el Hak, 2002). Differently from compliant surfaces, the discs are rigid and therefore more resistant to wear due to use. They may lead to further possibilities for optimized forcing conditions and controlled parametric studies than passive methods. In case of failure, the rotating discs would not lead to off-design drag increase, as for compliant surfaces because of undesired changes of mechanical properties, or for suction and ribletted surfaces because of dirt deposition, caused in the latter case by the very small maximum riblet height in flight conditions, about $0.15 \mathrm{~mm}$ (Viswanath, 2002). 
The influence of $R_{\tau}$ cannot be accounted for at this stage and is discussed in more detail in Ricco and Hahn (2013). The experimental verification of our simulations and studies on the effects of the flow geometry (free-stream vs. confined flows) and compressibility are other priorities in view of future applications. It is to be investigated how such variations impact on the drag reduction and the net power saved, and on the optimal tip velocity and diameter. It is our hope that further studies on this flow will follow to discern whether the performances in terms of drag reduction and net power saved can be improved.

\section{References}

U.M. Ascher, S.J. Ruuth, and B.T.R. Wetton. Implicit-explicit methods for time-dependent partial differential equations. SIAM J. Numer. Anal., 32:797-823, 1995.

K-S. Choi and B. R. Clayton. The mechanism of turbulent drag reduction with wall oscillation. Int. J. Heat Fluid Flow, 22:1-9, 2001.

K-S. Choi, J. R. DeBisschop, and B. R. Clayton. Turbulent boundary-layer control by means of spanwise-wall oscillation. AIAA J., 36(7):1157-1162, 1998.

L.G. Frechette, S.A. Jacobson, K.S. Breuer, F.F. Ehrich, R. Ghodssi, R. Khanna, C.W. Wong, X. Zhang, M.A. Schmidt, and A.H. Epstein. High-speed microfabricated silicon turbomachinery and fluid film bearings. J. Microel. Sys., 14(1):141-152, 2005.

K. Fukagata, K. Iwamoto, and N. Kasagi. Contribution of Reynolds stress distribution to the skin friction in wall-bounded flows. Phys. Fluids, 14(11):73-76, 2002.

M. Gad-el Hak. Compliant coatings for drag reduction. Progr. Aerosp. Sc., 38:77-99, 2002.

J. F. Gibson. Channelflow: a spectral Navier-Stokes simulator in C++. "http://www.channelflow.org/", 2006.

J. O. Hinze. Turbulence. McGraw Hill, Inc. - Second Edition, 1975.

W. J. Jung, N. Mangiavacchi, and R. Akhavan. Suppression of turbulence in wall-bounded flows by highfrequency spanwise oscillations. Phys. Fluids A, 4(8):1605-1607, 1992.

L. Keefe. A normal vorticity actuator for near-wall modification of turbulent shear flows. AIAA Paper, 97-0547, 1997.

L. Keefe. Method and apparatus for reducing the drag of flows over surfaces. United States Patent, 5,803,409, 1998.

L. Kleiser and U. Schumann. Treatment of incompressibility and boundary conditions in 3-D numerical spectral simulations of plane channel flows. In E. Hirschel, editor, Proc. 3rd GAMM Conf. Numerical Methods in Fluid Mechanics, pages 165-173. GAMM, Vieweg, 1980.

D. Kuang-Chen Liu, J. Friend, and L. Yeo. A brief review of actuation at the micro-scale using electrostatics, electromagnetics and piezoelectric ultrasonics. Acoust. Sci. \& Tech., 31:115 - 123, 2010.

S. B. Pope. Turbulent Flows. Cambridge University Press, 2000.

P. Ricco and S. Hahn. Turbulent drag reduction through rotating discs. Accepted in J. Fluid Mech., 2013.

P. Ricco, C. Ottonelli, Y. Hasegawa, and M. Quadrio. Changes in turbulent dissipation in a channel flow with oscillating walls. J. Fluid Mech., 700:77-104, 2012.

A.S. Sharma, J.F. Morrison, B.J. McKeon, D.J.N. Limebeer, W.H. Koberg, and S.J. Sherwin. Relaminarisation of $R e=100$ channel flow with globally stabilising linear feedback control. Phys. Fluids, 23(125105): $1-7,2011$.

C. Viotti, M. Quadrio, and P. Luchini. Streamwise oscillation of spanwise velocity at the wall of a channel for turbulent drag reduction. Phys. Fluids, 21(115109), 2009.

P.R. Viswanath. Aircraft viscous drag reduction using riblets. Progr. Aero. Sc., 38:571-600, 2002.

A.P. Willis, Y. Hwang, and C. Cossu. Optimally amplified large-scale streaks and drag reduction in turbulent pipe flow. Phys. Rev. E, 82(036321), 2010. 